山्ل FRANÇAISE

$\supset$ DE

묄 PÉDAGOGIE

\section{Revue française de pédagogie}

Recherches en éducation

$207 \mid 2020$

La LPPR et la réforme de l'enseignement supérieur et de la recherche: analyses critiques

BUISSON-FENET Hélène. Piloter les lycées. Le "tournant modernisateur » des années 1990 dans l'Éducation nationale

Grenoble : Presses universitaires de Grenoble, 2019, 264 p.

\title{
Sonia Revaz
}

\section{(2) OpenEdition}

\section{Journals}

Édition électronique

URL : https://journals.openedition.org/rfp/9356

DOI : $10.4000 /$ rfp.9356

ISSN : 2105-2913

Éditeur

ENS Éditions

Édition imprimée

Date de publication : 30 juin 2020

Pagination : 147-148

ISBN : 979-10-362-0297-1

ISSN : 0556-7807

Référence électronique

Sonia Revaz, «BUISSON-FENET Hélène. Piloter les lycées. Le «tournant modernisateur » des années 1990 dans l'Éducation nationale », Revue française de pédagogie [En ligne], 207 | 2020, mis en ligne le 30 juin 2020, consulté le 14 janvier 2023. URL : http://journals.openedition.org/rfp/9356 ; DOI : https://doi.org/10.4000/rfp.9356

Ce document a été généré automatiquement le 14 janvier 2023.

Tous droits réservés 


\section{BUISSON-FENET Hélène. Piloter les lycées. Le "tournant modernisateur" des années 1990 dans l'Éducation nationale}

Grenoble : Presses universitaires de Grenoble, 2019, 264 p.

\section{Sonia Revaz}

\section{RÉFÉRENCE}

BUISSON-FENET Hélène. Piloter les lycées. Le « tournant modernisateur » des années 1990 dans l'Éducation nationale. Grenoble : Presses universitaires de Grenoble, 2019, 264 p.

1 Dans Piloter les lycées, Hélène Buisson-Fenet se prête à l'exercice inédit de passer en revue les étapes de l'évolution de l'administration scolaire en France depuis les années 1990. Elle dépasse la description des outils de l'action publique pour questionner plus avant trois changements organisationnels qui ont marqué l'éducation française des trente dernières années : la référence à l'usager, l'instrumentation et la territorialisation. Le postulat central consiste à dire que ces trois changements sont au cœur d'un «tournant modernisateur» de l'action publique de l'Éducation nationale. À travers ce projet rétrospectif, l'autrice dessine les contours de cette modernisation en illustrant la façon dont elle s'est concrétisée dans différents contextes locaux.

Dans cet ouvrage, Buisson-Fenet se situe pleinement dans une sociologie politique de l'administration et de l'action publique de l'éducation; elle place l'action gouvernementale non seulement au centre de l'institution scolaire mais également au centre de la société. Les analyses sont construites sur un équilibre judicieux entre un intérêt pour les acteurs du "milieu de la hiérarchie" et la considération des institutions qui les entourent. En d'autres termes, ce sont les coulisses du gouvernement scolaire qui sont examinées. La diversité des configurations dans 
lesquelles les trois orientations modernisatrices se sont objectivées s'appréhende à travers onze études de cas réalisées à partir d'enquêtes effectuées dans différents départements, tantôt par l'autrice elle-même, tantôt par des confrères. L'hétérogénéité des cas est dévoilée à travers trois méthodes principales: analyses de documents produits par les services administratifs étudiés, entretiens semi-directifs et observation. L'ouvrage compte trois parties divisées pareillement en quatre chapitres et consacrées, chacune, à l'un des trois changements du tournant modernisateur.

3 La partie 1 traite de la référence aux usagers, de leur identité et de l'évolution de leur place dans l'administration scolaire. Dans le chapitre 2, l'autrice évoque notamment le développement de la relation école-famille. Elle l'illustre par une étude de cas réalisée dans trois lycées marseillais sur l'intervention des parents dans l'orientation de leurs enfants. Elle aboutit, entre autres résultats, au constat d'un pouvoir de décision peu partagé entre les familles et les chefs d'établissement, dont le monopole participe à la ténacité des « régularités institutionnelles ». En guise de prolongement de la réflexion sur l'usager, le chapitre 4 aborde sa relation avec l'enseignant et met en évidence, à travers l'analyse du développement professionnel de l'auxiliariat de vie scolaire (AVS) dans l'académie de Lyon, la façon dont leurs nouveaux rapports ont profondément modifié les pratiques enseignantes.

4 La partie 2, particulièrement intéressante, est celle qui marque l'inscription des trois changements dans une logique de continuité puisqu'elle introduit l'instrumentation comme prolongement du rôle grandissant de l'usager et comme condition de la territorialisation. En traitant de la décentralisation de la gestion de l'école et du passage d'un État interventionniste à un État régulateur, elle pose la question des outils - fournis non seulement aux familles mais aussi au personnel administratif et enseignant - qui ont permis cette évolution. Trois types d'instruments sont discutés : des applications informatiques, des équipements pour évaluer et contractualiser les établissements et des cadres budgétaires institutionnalisés.

5 Le chapitre 6 rappelle le rôle grandissant des usagers et aborde la façon dont les applications informatiques ont contribué à les rendre plus actifs dans les processus d'orientation scolaire. L'étude de cas présentée propose de revenir sur l'utilisation du logiciel PAM (pré-affectation automatique multidossiers), élaboré dans le but de prendre en considération les préférences des usagers et, parallèlement, améliorer la mixité des établissements scolaires mise à mal par les contournements à la carte scolaire. L'étude de cas concerne la première utilisation du logiciel dans les lycées professionnels d'Aix-Marseille en 2002 et se focalise sur le personnel des établissements, dont les tâches ont été modifiées et augmentées en raison de nouvelles responsabilités liées à l'affectation des étudiants. En faisant résonner le dispositif PAM à l'outil Admission post-Bac (APB) mis en place une année plus tard dans le but de rationaliser le recrutement dans les universités, on comprend « non seulement [que ces outils] mettraient en scène un usager scolaire rationnel qui n'existe pas [...] ; mais [que] les publics seraient alors mobilisés pour matérialiser des décisions déjà prises " (p. 108). La "rhétorique de l'usager» serait alors outrepassée par la traduction administrative des injonctions à la modernisation.

6 La partie 2 se termine sur la double perspective qu'offre l'étude des instruments: pensés tantôt comme des indicateurs de référentiels - ils renseignent des cadres cognitifs et normatifs qui structurent l'action publique - tantôt comme des agents autonomes capables d'orienter l'action publique à travers la responsabilisation et 
l'incitation des usagers à l'auto-contrôle. La synthèse des différents modes d'instrumentation montre qu'à travers les processus de traduction - indispensables à l'appropriation des instruments -, les pratiques du personnel de l'administration scolaire se définissent dans une logique a posteriori. En somme, Buisson-Fenet montre que les instruments n'augurent que partiellement leur définition et qu'ils sont façonnés progressivement. C'est ce qu'elle a montré par exemple à travers l'étude de cas 10 qui met en évidence la normalisation graduelle des pratiques professionnelles des gestionnaires et agents comptables de l'Éducation nationale, dont le pouvoir décisionnaire s'est accru suite à la réforme du cadre comptable et budgétaire (RCBC) des établissements publics locaux d'enseignement (EPLE).

7 Après les rhétoriques de l'usager et des instruments, c'est celle du territoire qui est traitée dans la partie 3 ; Buisson-Fenet parle également d'un «ordre local de l'école ». Le chapitre 9 revient sur la naissance de l'intérêt des sociologues pour les différences locales qui marquent l'Éducation nationale et propose, par ailleurs, de clarifier la définition de "territoire » dans une perspective de sociologie de l'action publique de l'éducation. Sur ces bases, l'autrice entame les chapitres 10 et 11 avec une réflexion sur la décentralisation et met en évidence la centralité des processus de négociation dans les rapports entre l'État et les localités. Les deux études de cas qui y sont exposées montrent comment les arrangements entre les deux parties aboutissent à des compromis locaux très divers. En particulier, l'étude de cas tirée du projet KnowPol (Knowledge \& Policy, 2006-2011) coordonné par Delvaux et Mangez sur la transition formation-emploi met en évidence la nature locale des référentiels et la proéminence de l'influence de l'État. Des nuances sont toutefois apportées quant à ces logiques d'action: le pouvoir normatif de l'État sur les questions de formation professionnelle est pondéré par les organisations professionnelles, groupes de pilotage et autres collectifs locaux. Bien qu'elles aboutissent à des compromis, les négociations occasionnent diverses tensions inter-institutionnelles qui touchent notamment aux calendriers, contraintes financières et procédures gestionnaires.

L'ouvrage se termine par une conclusion sur les apports des études de cas recensées. En remobilisant l'objectif de l'ouvrage qui était de penser la façon dont l'administration de l'Éducation nationale s'est modernisée, l'autrice clôt sa réflexion sur l'évolution incrémentale de l'administration scolaire: «le poids des variables politiques dans ce secteur d'action publique favorise le statu quo» (p. 227). En définitive, par-delà le panorama des changements dont le gouvernement scolaire a fait l'objet ces trente dernières années, Piloter les lycées apporte beaucoup à la compréhension des implications des changements institutionnels pour les acteurs du milieu de la hiérarchie. Si l'objectif d'éclairer le changement dans l'action publique de l'éducation en France est clair, on peut toutefois regretter l'aspect secondaire du raisonnement sur l'influence de sources internationales. Pourtant, la démonstration de Buisson-Fenet est tout à fait convaincante et cette brève recension ne rend pas justice à la richesse de ses réflexions et à la diversité des études de cas qu'elle a développées. 


\section{AUTEURS}

SONIA REVAZ

Université de Genève, Groupe genevois d'analyse des politiques éducatives 